\title{
Migrations et relations internationales
}

Les apories de la gestion multilatérale des migrations internationales?

Migration and international relations. The pitfalls of managing international migration multilaterally

\section{Hélène Thiollet}

\section{OpenEdition Journals}

Édition électronique

URL : http://journals.openedition.org/transcontinentales/787

DOI : 10.4000/transcontinentales.787

ISBN : 978-2-7351-1557-0

ISSN : $1775-397 X$

Éditeur

Editions de la maison des sciences de l'homme

Édition imprimée

Date de publication : 31 décembre 2010

ISSN : 1950-1684

Référence électronique

Hélène Thiollet, « Migrations et relations internationales », Transcontinentales [En ligne], 8/9 | 2010, document 2, mis en ligne le 31 décembre 2010, consulté le 08 septembre 2020. URL : http:// journals.openedition.org/transcontinentales/787 ; DOI : https://doi.org/10.4000/transcontinentales. 787

Ce document a été généré automatiquement le 8 septembre 2020

Tous droits réservés 


\title{
Migrations et relations internationales
}

\author{
Les apories de la gestion multilatérale des migrations internationales? \\ Migration and international relations. The pitfalls of managing international \\ migration multilaterally
}

Hélène Thiollet

1 À l'occasion du Dialogue de haut niveau sur les migrations organisé à New York le 15 septembre 2006, les organisations multilatérales au premier rang desquelles l'oNU avaient cru s'emparer de la question des migrations internationales. Le Dialogue avait été préparé de longue date avec le lancement en 2001 d'une vaste consultation sur les migrations par le secrétaire général Kofi Annan et la création d'une Commission globale sur les migrations internationales ${ }^{1}$. Point d'orgue de cette consultation, l'événement voté en 2003 par l'Assemblée générale ${ }^{2}$ devait permettre à l'O NU de reprendre la main face aux organisations économiques internationales (Banque mondiale, Fonds monétaire international, Organisation mondiale du commerce) dans l'énonciation des grandes tendances d'une politique publique des migrations internationales. Le Dialogue avait mobilisé l'Assemblée générale de l'onu, des experts d'organisations internationales gouvernementales - comme l'Organisation internationale des migrations - et non gouvernementales, des groupes de travail incluant des représentants des grands pays d'immigration et d'émigration, et avait impliqué les agences onusiennes (PNUD, UNICEF, UNESCO, etc.) qui avaient toutes lancé des programmes spécifiques liant leurs secteurs d'activité à la migration. L'association des thèmes migrations et développement devait permettre à l'ONU d'attirer l'attention des États là où le discours sur la protection des droits des migrants a échoué. L'ONU devait en outre proposer une architecture politique globale et multidimensionnelle de gestion des migrations internationales. Les différentes conférences accompagnant l'ouverture de la soixante et unième session de l'Assemblée générale ont largement réaffirmé que les migrations sont un jeu où tous sont gagnants et que l'ONu était le partenaire naturel de l'élaboration des politiques migratoires à l'échelle nationale et 
régionale. Mais, à l'heure où les pays occidentaux, États-Unis et France en tête, réaffirment la « souveraineté » de l'État - celui du pays d'accueil particulièrement - sur la gestion des migrations, quel est l'avenir de cette louable et difficile tentative de multilatéralisation des politiques migratoires?

\section{L'échec de l'approche juridique et sociale}

2 La Convention sur les droits des travailleurs migrants et de leur familles votée en 1990 n'a pu entrer en vigueur que le $1^{\text {er }}$ juillet 2003, lorsqu'elle a atteint le seuil minimal de 20 ratifications. L'échec cuisant de ce texte mesure montre la faiblesse de la pression onusienne sur la question des migrations et augure mal de l'avenir du magistère politique de l'ONU sur la gestion des migrations internationales ${ }^{3}$.

Il faut dire que le principe de la convention est audacieux. Il consacre la nondiscrimination entre nationaux et travailleurs migrants dans l'application des droits humains en général et dans le respect de droits spécifiques. Il mentionne le droit des migrants de former des associations et des syndicats (Art. 40), de prendre part aux affaires publiques et aux élections de leur État d'origine (Art. 41), de vivre en famille (Art. 44), les droits de l'enfant à un nom, à l'enregistrement de sa naissance, à une nationalité, à l'accès à l'éducation (Art. 29, 30), la prohibition d'expulsion collective (Art. 22), etc. Il promeut la non-discrimination entre travailleurs en situation régulière ou irrégulière et, partant, fait du travailleur migrant, quel que soit son statut juridique, un sujet du droit. Résidents, travailleurs étrangers sous contrat, immigrés clandestins ne sont plus définis par leur statut administratif dans le pays d'accueil mais par leur situation de travailleurs migrants. L'ethos migratoire prime sur le statut individuel et la mobilité elle-même devient, avec ce texte, un critère de qualification juridique ${ }^{4}$.

4 La convention va plus loin : elle identifie non seulement le travailleur mais aussi sa famille comme sujet des droits énumérés. Le texte de 1990 fait de la mobilité le critère d'accès à un régime de protection international et reprend les termes de conventions antérieures (droits de l'homme, droits de l'enfant, droits de la femme, droits des travailleurs) pour les appliquer à une catégorie nouvelle. Et la définition de cette catégorie (migrant légal ou clandestin) fait justement le caractère révolutionnaire d'un texte qui remet en cause la souveraineté de l'État sur le territoire et les citoyens au profit d'une lecture trans-nationale des droits des individus quel que soit leur statut (travailleurs migrants et leurs familles).

\section{Migration et développement : les succès de l'approche économique?}

5 Le lien entre migration et développement est au cœur des réflexions de l'ONU: la migration et les flux financiers qu'elle génère (remises migratoires) sont-ils un adjuvant ou un obstacle au développement des pays les plus pauvres? Deux questions cristallisent le débat : le brain drain (ou brain gain, selon le point de vue) et les remises migratoires. Les enjeux économiques de la migration se déclinent en termes de capital humain et de capital financier ${ }^{5}$.

6

Alors qu'elle développe depuis plusieurs années un agenda liant aux migrations et programmes de micro-finance les risques et les bénéfices de la migration et des remises 
migratoires, la Banque mondiale refuse de considérer ces sources privées comme des substituts aux politiques de développement menées par les États, les estimant bien comme un complément de celles-ci. Cette précaution n'est pas sans importance. Les remises migratoires relèvent des finances privées, souvent via des canaux informels, tandis que le développement est « une affaire d'État " (qu'il s'agisse de l'allocation des financements ou des plans de développement). Sur les remises migratoires, les États n'ont longtemps eu comme levier d'action que la manipulation des taux de change et des frais de transferts par le secteur bancaire officiel. Ces prélèvements et le coût des intermédiaires financiers dans le contexte de pays souvent marqués par la corruption et les incertitudes financières expliquent en partie l'importance des transferts informels.

7 En 2004, les remises enregistrées étaient supérieures à l'investissement privé et public dans 36 pays en développement, elles dépassaient en valeur les exportations les plus importantes dans 28 pays. En 2006, elles étaient plus importantes que les revenus du tourisme au Maroc et de l'exportation du thé au Sri Lanka, et elles représentaient plus de $35 \%$ du produit intérieur brut (PIB) de la Moldavie, du Tadjikistan et du Tonga, $25 \%$ de celui du Lesotho ${ }^{6}, 23 \%$ de celui du Liban, de la Jordanie et d'Haïti. Au-delà de ces aspects quantitatifs, les flux de remises étaient considérés comme moins dépendants des cycles économiques que d'autres sources de revenus et représentaient un facteur de stabilité en période de crise financière (comme durant la crise en Thaillande et en Indonésie en 1998). La crise financière de 2008 a certes entrâné une diminution des revenus et la chute des investissements et de l'activité à l'échelle globale. Elle est essentiellement due à la diminution des flux migratoires liée à des politiques antiimmigration des pays riches et aux fluctuations des taux de change ${ }^{7}$. En Asie du Sud, comme en Afrique et au Moyen-Orient, les remise ont augmenté en 2008 même si leur croissance a été moins forte que prévue.

En bref, les remises migratoires sont une manne financière deux fois plus importante que l'aide au développement consentie par les pays développés ${ }^{8}$ et elle attire la convoitise des États. L'enjeu est de taille pour l'organisation intergouvernementale qu'est l'ONU : les gouvernements tentent de revenir sur le caractère privé de la rente migratoire, avec pour appui théorique, par exemple, la proposition de Jagdish Bhagwati en 1976, taxing the brain drain, qui permettait de résoudre le dilemme du brain drain et des remises migratoires d'un coup de politique fiscale?.

\section{Régionalisation ou internationalisation?}

9 Les migrations touchent 224 millions de personnes à l'échelle de la planète mais les manifestations du phénomène sont inégales, dépendantes de facteurs économiques et sociaux discernables à l'échelle individuelle, nationale, régionale et internationale. Vus de Sirius, les taux d'émigration les plus fort sont à chercher dans les pays à revenus intermédiaires (3,3\% de migrants en 2006 contre $2,9 \%$ dans les pays les moins avancés $[\mathrm{PMA}]^{10}$ ). Selon une loi bien connue des sociologues de la migration, bouger demande des ressources, financières, humaines, informationnelles, et ce ne sont pas les plus démunis qui parviennent à migrer. À l'échelle internationale, les pays à revenu intermédiaire et les pays de l'Organisation de coopération et de développement économique (OCDE) à fort revenus montrent les taux de mobilité internationale les plus élevés. 
10 Les migrations sont majoritairement des phénomènes intrarégionaux. Le nombre de migrants qui partent d'Afrique est bien moins important que celui des migrants qui circulent en Afrique, ou en Asie. Les taux, mais aussi l'historicité de ces flux intrarégionaux (et des diasporas qui en résultent), sont un facteur souvent oblitéré par la prégnance des modèles de migrations économiques Nord-Sud (vers l'Europe ou les pays occidentaux d'immigration, les États-Unis, le Canada, l'Australie).

11 Les débats des différents bureaux régionaux du Conseil économique et social de l'oNU reflètent les différences entre les processus d'intégration régionale. Sur la question du rôle des remises migratoires dans le développement, l'Afrique prône une approche intergouvernementale résolument étatique portée par le Nouveau partenariat pour le développement de l'Afrique (NEPAD), l'Union africaine et les groupements infrarégionaux (Communauté économique des États de l'Afrique de l'Ouest [CEDEAO], la Communauté de développement d'Afrique australe [SADC], etc.) : les États doivent être partie prenante dans la gestion des migrations et de l'argent des migrants pour en faire une source de développement équilibrée et productive. L'Asie partage les analyses de la Banque mondiale en matière de libéralisation des flux financiers, d'abaissement des coûts de transfert de remises, et de transparence des institutions bancaires. Le désengagement de l'État n'est modéré que par la nécessité de trouver des moyens de protéger les travailleurs asiatiques à l'étranger (particulièrement les femmes), et notamment dans le Golfe.

12 Les échelles de gestion régionale et internationale sont transitives: les migrations peuvent comporter plusieurs étapes, ne sont pas nécessairement linéaires, et le politique participe à la complication des schémas migratoires. En Afrique australe, l'émigration des personnes qualifiées, notamment le brain drain médical qui touche la Zambie et le Zimbabwe, a donné lieu à un accord en $2006^{11}$, interdisant aux pays les plus riches de la région (l'Afrique du Sud et le Nigeria) de débaucher le personnel médical des autres pays. Mais les médecins et les infirmières zambiens et zimbabwéens ont déjà prévenu que ce type de pratiques risquait d'augmenter l'émigration vers la GrandeBretagne. La coercition est rarement efficace, qu'il s'agisse de fermer les frontières pour empêcher d'entrer ou de partir.

13 L'espace de libre circulation organisé par la CEDEAO a constitué une réponse et les migrations économiques intra-africaines se sont développées avec l'essor de certaines sous-régions (par exemple, les Nigérians au Burkina Faso). Mais, ce type de réponse n'est envisageable que dans un cadre coopératif et dans un contexte de croissance économique...

14 La diffusion des «meilleures pratiques" semble compromise par la spécificité des contextes régionaux. Le politique revient encore et toujours dans le champ de mire des négociateurs et des experts de l'ONU. L'exemple de l'Afrique illustre largement les obstacles que rencontre une gestion consensuelle et multilatérale des flux de personnes. Si une grande partie des pays africains semble «ne pas avoir de politique migratoire ${ }^{12}$, les migrations sont, particulièrement en Afrique, un enjeu de sécurité nationale, régionale. Avec $14 \%$ de la population de la planète, l'Afrique comptait $33 \%$ des réfugiés reconnus par le Haut commissariat aux réfugiés (HCR) en 2005 et $20 \%$ fin $2008^{13}$; on dénombre plus de 17 millions de migrants sur le continent. Migration et flux de réfugiés sont difficiles à comptabiliser en Afrique comme ailleurs. Pourtant, la démographie et les mouvements de populations sont un élément clé des conflits étatiques ou infra-étatiques et de la politique régionale à l'échelle infrarégionale, 
comme le démontrent encore aujourd'hui la Corne de l'Afrique et l'Afrique centrale ${ }^{14}$. Instrumentalisés dans les conflits, enjeux de luttes politiques au niveau national et international, les réfugiés et les migrants participent à la stabilisation et à la transnationalisation du politique. À l'échelle mondiale, la mobilité est un produit de la violence autant que des crises économiques, des conditions environnementales, mais le contexte politique la rend « durable». En Afrique, quelques Eldorado peuvent accueillir les circulations transfrontalières (l'Afrique du Sud, le Nigeria...) mais les ressources du continent restent minces. La permanence de conflits provoque la mobilité et hypothèque sa résorption: la question de la gouvernance supranationale des migrations - au niveau régional ou international - est intimement liée à la résolution des conflits qui touchent le continent.

\section{Conclusion}

La Commission globale sur les migrations de l'ONU affirmait en 2005 que les possibilités et les limites de l'intervention de l'État en matière migratoire devenaient de plus en plus claires, mais le texte restait très flou et précautionneux quant aux moyens réels de pression et d'action des organisations internationales ou régionales sur la politique des États ${ }^{15}$. À observer les murs et autres dispositifs de contrôle se multiplier sur les frontières américano-mexicaine, israélo-palestiniennes, yéméno-saoudiennes, à étudier les textes de lois récemment adoptés sur l'immigration ou la nationalité en France comme ailleurs, on peine à distinguer les marques d'une quelconque multilatéralisation de la question migratoire. Les partenariats noués entre les pays du Nord et du Sud sur les questions de circulation des personnes ne font que confirmer la nature bilatérale et assurément inégale du processus d'élaboration des politiques migratoires.

Au-delà du partage d'informations, d'expériences, entre experts internationaux des différentes organisations, délégués régionaux ou spécialistes, le but du Dialogue de haut niveau est de convaincre les États que la coopération internationale est le meilleur outil de maximisation des profits de la migration. De cela, les 34 États signataires de la convention de 1990 - tous des pays dits « du Sud»- sont convaincus, mais les grands pays d'immigrations sont loin de l'être...

\section{NOTES}

1. Franck Petiteville et Andy Smith, "Analyser les politiques publiques internationales ", Revue française de science politique, vol. 56, 3/2006 : 357-366. URL : www.cairn.info/revue-francaise-descience-politique-2006-3-page-357.htm.

2. Dans sa résolution 58/208 du 23 décembre 2003, l'Assemblée générale de l'oNu a décidé de consacrer un dialogue de haut niveau aux migrations internationales et au développement. La discussion devait permettre d'identifier les moyens de maximiser les bénéfices de la migration et de minimiser ses impacts négatifs sur les pays d'origines comme sur les pays d'accueil. Le 
dialogue devait avoir une orientation nettement politique dans la ligne des objectifs du millénaire.

3. http://untreaty.un.org/French/TreatyEvent2001/pdf/12f.pdf. Convention votée le 18 décembre 1990. En 2009, la Convention a été ratifiée par 44 États parties.

4. Les progrès d'un ordre juridique international fondé sur le respect de la personne et de ses droits avaient déjà permis l'avènement d'une forme d'indifférenciation entre citoyens et noncitoyens : c'est le fait d'être une femme, un enfant, un travailleur, et non la citoyenneté, qui fait de l'individu un sujet du droit (droit de l'homme, de l'enfant, de la femme, droit du travail etc.).

5. Plus précisément, la migration de travailleurs qualifiés est-elle une perte ou un gain pour les pays d'origine, et l'argent que les émigrés renvoient chez eux est-il un bien ou un mal pour le développement de ces mêmes pays?

6. Banque mondiale, Global Economic Prospects, 2007.

7. "Migration and Remittance Trends 2009. A better-than-expected outcome so far, but significant risks ahead ", Migration and Development Brief, $\mathrm{n}^{\circ}$ 11, Banque mondiale, 3 novembre 2009.

8. Devant les remises et l'aide internationale, la première source de fonds des pays en développement est l'Investissement direct étranger (IDE) : en 2003, les pays en développement ont reçu en tout 130 milliards de dollars d'IDE et environ 75 milliards de remises migratoires. Richard H. Adams Jr., "Migrations, remittances and development: The critical nexus in the Middle East and North Africa", Nations unies, 18 avril 2006: http://www.un.org/esa/ population/publications/EGM_Ittmig_Arab/P01_Adams.pdf [site visité le 22 novembre 2006].

9. Jagdish Bhagwati et John Wilson, Migration and Income Taxation, Cambridge, MIT Press, 1991.

10. Recueil de statistiques 2008 sur les migrations et les envois de fonds, Banque mondiale.

11. International Migrations and Development: Implications for Africa,Nations unies, Economic commission for Africa, $2006: 36$.

12. ONU Division de la population, Rapport sur les migrations internationales, 2000. L'ONU y évalue les politiques migratoires et le degré de satisfaction des États vis-à-vis des leurs flux migratoires. Voir aussi, Hania Zlotnic, "Migrants' rights, forced migrations and migration policy in Africa »,Conference paper, 2003 : http://pum.princeton.edu/pumconference/papers/6Zlotnik.pdf.

13. Source : Agence des Nations unies pour les réfugiés (UNHCR), 2010.

14. Pour ne prendre qu'un exemple, les déplacés et les réfugiés du Darfour sont, depuis 2003, au cœur de la dynamique du conflit tchado-soudanais et des logiques de guerres civiles soudanaises. En 2004, le Tchad a reçu plus de 131000 réfugiés soudanais alors que le pays lutte pour assurer la survie d'une partie de sa population.

Les populations déplacées peuvent être victimes et otage des diplomaties, instruments de négociation avec les organisations internationales et les organisations non-gouvernementales (ONG).

15. Les migrations dans un monde interconnecté, nouvelles perspectives d'action, rapport de la Commission globale sur les migrations internationales, $2005:$ http://www.gcim.org/mm/File/ French.pdf [site visité le 22 novembre 2006] 


\section{RÉSUMÉS}

Les migrations internationales sont aujourd'hui un des enjeux majeurs de la gouvernance globale. Elles restent pourtant l'objet de politiques régaliennes où dominent les accords bilatéraux sans que s'impose un régime de gestion multilatéral. Les migrations sont une des pierres d'achoppement du multilatéralisme et leur gestion est revendiquée par les États comme une dimension inaliénable de leur souveraineté (économique, territoriale...). Les institutions multilatérales tentent de proposer des modalités de gouvernance multilatérale des migrations comme phénomène social global. À l'oNU, le Haut commissariat aux réfugiés (HCR) s'occupe des réfugiés et l'Organisation internationale du travail (огт) des migrants en leur qualité de travailleurs. La Banque mondiale et le FMI sont particulièrement actifs sur la question des migrations et des transferts financiers qui les accompagnent alors que l'UNICEF s'intéresse aux conséquences sociales de la mobilité sur les familles dans les pays d'origine ou d'accueil. L'Organisation internationale des migrations travaille quant à elle en marge de l'oNU. L'onu n'est pas parvenu à imposer la Convention sur les droits des migrants de 1990 et ne parvient pas à faire émerger une ligne d'action collective dans l'arène multilatérale sur la question migratoire. Pourtant, dans la sphère onusienne et au-delà, les organisations internationales négocient l'émergence d'une agence spécialisée dans les migrations. À partir de la stratégie historique du $\mathrm{HCR}$ dans le champ multilatéral et d'une innovation juridique récente - le concept de «migrations mixtes »-, on décrit une des tentatives de gestion juridique de la mobilité forcée et volontaire. En 2006, le concept émerge avec l'expérience des migrations sub-sahariennes à travers la Méditerranée. On s'intéresse ici à son utilisation dans le cas de migrations depuis la corne de l'Afrique vers le Yémen. Le concept de "migrations mixtes » permet d'observer les prémisses de la remise en question de la partition entre réfugiés et migrants économiques. Cette analyse nous permet de mettre en lumière les apories contemporaines des conditions juridiques et statutaires de la mobilité et les réponses pragmatiques qui y sont apportées. À partir de sa compétence de terrain sur les populations réfugiées et d'innovation organisationnelles récentes, le HCR se positionne dans le champ politique international comme gestionnaire potentiel de la mobilité internationale face à l'OıT ou à une agence non-onusienne comme l'OıM.

Today, one of the biggest issues facing global governance is international migration. It is nevertheless dominated by national policy and bilateral agreements, and lacks a multilateral management system. Indeed, migration is one of the stumbling blocks of multilateralism, because most countries consider migration management is key to national sovereignty (in economic and territorial terms). Multilateral institutions attempt to suggest multilateral migration governance mechanisms as a global social phenomenon. At the UN, the High Commission on Refugees (UNHCR) was created to deal with refugee issues, and the International Labour Organisation (ILO) for migrant workers. The World Bank and the International Monetary Fund (IMF) are very active in issues affecting migrants and remittances, while UNICEF focuses on the social consequences of this mobility on families in home and host countries. The International Organisation for Migration (IOM) works on the fringes of the UN. The UN was unable to impose the Convention on the Rights of Migrants in 1990, and has had difficulty in developing a joint programme of action for the multilateral management of migration. Nevertheless, on the UN level and above, international organisations are negotiating the creation of an agency specialising in migration. Using the HCR's past multilateral strategy and a recent legal innovation - the concept of mixed migration - we describe a legal attempt to manage forced and voluntary mobility. "Mixed migration" first appeared in 2006 to describe Sub-Saharan migration through the Mediterranean region. We examine how it is applied to migrant flows 
from the Horn of Africa to Yemen. The concept of mixed migration can be used to observe the ways in which the difference between refugees and economic migrants are being broken down. This highlights the pitfalls in the legal and statutory conditions affecting mobility, and the practical solutions put forward to overcome this situation. With its experience in dealing with refugee populations and recent organisational innovations, the UNHCR seems better placed than the ILO or the IOM to manage international mobility in the global political context.

\section{INDEX}

Keywords : HCR, hign devel dialogue, migrants workers, migration, mobility, UNO

Mots-clés : dialogue de haut niveau, flux migratoires, HCR, mobilité, ONU, travailleurs migrants Thèmes : migration, mobilité

\section{AUTEUR}

\section{HÉLÈNE THIOLLET}

École des affaires internationales (Sciences Po), Maison française d'Oxford (MFO-UMIFRE 11, http://www.mfo.ac.uk/fr). Elle a été chercheur invitée au Centre français pour l'archéologie et les sciences sociales (CEFAS-UMIFRE 5, http://www.cefas.com.ye) à Sana'a, Yémen en 2005, et a participé de 2007 à 2010 au projet Anr « Golfe arabo-persique et Europe : violences et contre-violences », sous la direction de François Burgat (IFPO, Beyrouth-Ireman, Aix-en-Provence) helene.thiollet@sciences-po.fr 